\title{
Biomechanical aspects of dynamic stability
}

\author{
G. Meyer • M. Ayalon
}

Published online: 15 March 2006

(C) EGREPA 2006

\begin{abstract}
Walking is a fundamental motor skill that significantly affects the level of independence in older adults. The amount of variability present in a walking pattern reflects the quality of neuromuscular control. It is well established that a large proportion of falls in older people occurs when walking. The prevention of falls is vital for minimizing disability, preventing injury, and impeding the development of frailty and subsequent deterioration in quality of life. The present literature review focuses on dynamic stability. In dynamic stability, both the base of support and the center of mass are in motion, and effective balance function is required. In general, older adults are expected to have different movement patterns from younger adults, expressed by differences in limb kinematics and kinetics. A better understanding of the biomechanical variables involved can help anticipate and prevent potential falls.
\end{abstract}

Keywords Balance · Gait · Falls

\section{Introduction}

Walking is a fundamental motor skill that significantly affects the level of independence in older adults. There is no unique walking pattern, and the pattern varies

\footnotetext{
G. Meyer $\cdot$ M. Ayalon $(\varangle)$

Biomechanics Laboratory,

Zinman College of Physical Education and Sport Sciences,

Wingate 42902, Israel

e-mail: ayalon-m@wincol.ac.il

G. Meyer

e-mail: ganit20@hotmail.com
}

from person to person. The amount of variability present in a walking pattern reflects the quality of neuromuscular control. Minor variability indicates better neuromuscular control and walking stability. The walking pattern of individuals becomes more inconsistent as they grow older, and walking patterns are considered stable until there is evidence of a fall. It is well established that a large proportion of falls in older people occurs when walking. The prevention of falls is vital for minimizing disability, preventing injury, and impeding the development of frailty and subsequent deterioration in quality of life [39].

Falls result from a complex interaction of risk factors, and over 130 of these risk factors have been identified $[14,40]$. Effective control of balance depends on the interaction of many factors including integration of visual, vestibular, and proprioceptive information concerning body position, appropriate biomechanical alignment, sufficient muscle strength, and quick, coordinated muscle activation patterns. Impairment in any of these domains reduces an individual's ability to balance the multiple connections in the musculoskeletal system while standing or ambulating.

Posture is the term used to describe the orientation of any body segment relative to the gravitational vector. Balance refers to body posture dynamics that prevent falling [53]. To maintain balance, the postural control system keeps the body's center of mass (COM) over the base of support (BOS). The BOS is the minimum area enclosing the body's contact with the ground. Therefore, while standing, the BOS is the area enclosing the soles of the feet (or shoes). A smaller BOS gives a smaller area for the alignment of the $\mathrm{COM}$, and the body in such a position is considered less stable. This postural control process is referred to 
as dynamic stability. From a mechanical point of view, the goal is to regulate the relationship between the $\mathrm{COM}$ and the BOS. Situations requiring balance can be classified into three general conditions: maintenance of a stable position, postural adjustment to voluntary movements, and reactions to external predicted and unpredicted perturbations (slipping or tripping). In dynamic stability, both the BOS and the $\mathrm{COM}$ are in motion. Prevention of falls requires effective balance function under dynamic conditions because most falls are caused by sudden motion of the BOS or by sudden acceleration of the COM. Stability can be defined as the ability of a system to return to its original state, i.e., desired movement trajectory after a disturbance. Many elderly individuals possess poor dynamic stability which can increasingly worsen as a result of a combination of chronic deterioration of musculoskeletal, neuromuscular, and somatosensory systems, due to genetic traits, lifestyle, and other factors $[25,26,43,44,51]$. The performance of different daily life activities has a threshold level. In many cases, older adults closely meet that threshold with little reserve effort. Therefore, every exceptional movement which exceeds that threshold can lead to an inability to perform simple tasks such as recovering from a light perturbation in walking.

\section{Review of literature}

\section{Gait characteristic}

Older adults tend to walk more slowly (self-selected and maximal speed), have a shorter step length, broader walking base, less vertical displacement of the COM, and disturbed coordination between upper and lower extremities compared to young adults. This results in a gait cycle with a longer stance and/or longer double support time. It is generally believed that the changes in temporal gait parameters and body kinematics of older adults is a more stable or safer gait pattern, suggesting that older people compensate for their reduced physical capabilities by being more cautious $[3,4,8-10,16,18-21,24,27,28,32,34,39$, $41,42,47,50,54,55]$.

Although gait changes could represent the adoption of a more stable walking pattern among the elderly, it has been suggested that these same safety adjustments may constitute risk factors for falls [26]. It appears that reduced walking speed is a compensatory strategy to maintain balance. Nevertheless, the fact that some older people who adopt this strategy are still likely to suffer from falls suggests that there is another aspect in their gait predisposing them to losing balance when walking [26].

\section{Dynamic stability}

The key to dynamic stability is the momentum control of the COM. The distribution of body mass is such that two-thirds of mass is in the head, arms, and trunk. Because of the large mass and inertia moment of the upper body, its position and movement (forward momentum) can be critical in the overall stability of the upright stance [53]. Individual self-selected walking speed is optimal in relation to minimizing the variability of head and pelvic movements [26]. The use of momentum by older adults is perhaps a compensatory strategy. When there is insufficient lower extremity torque generating capacity, upper body momentum may be used to maintain the gait at a steady state [30, 31, 46, 52]. To assist the development of speed and movement, a large range of momentum generation is employed to compensate weak quadriceps [48]. In contrast, momentum must also be controlled and attenuated to maintain stability. The moment of force at the hip has two functions: one is to keep the lower limb from collapsing during the weight support phase, and the second is to help control the upper body position during weight transfer and the single limb phase. Older adults limit their gait velocity to lessen momentum generation. This is a result of insufficient balance control or strength needed to dissipate momentum generated by faster gait velocity. Age-related changes in lower trunk movement control during gait preserve upper body posture and walking speed. Such control requires a leading trunk and higher mechanical energy demands by the lower trunk's musculoskeletal system. These two factors may reduce the ability to recover from dynamic instabilities [37].

\section{Responses to perturbation}

Recovery from significant perturbation, such as a slip, can be successful when movement stability can be reestablished with protective stepping. An effective stepping response is often critical in avoiding a fall. Compensatory stepping, the act of taking a step from a stationary position or dynamic situation, requires the individual to in effect lose balance and then regain a stable posture once the step has been completed. Successful balance recovery by stepping is governed by a combination of step length, step execution time, and leg strength [17]. This activity generally becomes more difficult with advancing age. Stepping is necessary if there is sufficiently high velocity of the COM, 
even if the COM is located within the BOS at step initiation. Older adults employ a rapid stepping response compared to young adults under the same conditions because such stepping responses are less threatening to loss of balance $[29,38]$.

Proper initial foot placement for gait initiation and for stepping recovery responses should also be considered, and the first step length variability observed among elderly fallers may be an important predictor of postural problems. Gait initiation, which is a transient phase between standing and walking, could contribute to an increase in age-related variability in the control of foot placement (anterior-posterior and mediallateral) and/or COM displacement [35, 43].

Imbalance of the whole body during obstacle crossing may cause inappropriate movement of the lower extremities and result in foot-obstacle contact. Older adults seem to use a more conservative strategy for obstacle crossing. In a study comparing obstacle crossing of older and young adults, older adults demonstrated crossing velocity reductions of the leading limb, trailing limb, and COM compared to young adults [17, 23]. Older adults adopted a swing hip flexion strategy to achieve a higher leading toe clearance than young adults. With increasing obstacle height, the older adults increased leading toe clearance by changing fewer joint angular components. In contrast, when the trailing limb was crossing, the older group showed no significant difference in the trailing toe clearance compared to the young group, although different joint kinematic patterns were evident [33]. The angular velocity of hip flexion from toe off to toe over the obstacle in older adults was $20 \%$ less than that in young adults [15]. We now know that older adults demonstrate shorter crossing step lengths, slower crossing velocities, and shorter postobstacle heel strike distances [6]. Longer pre-obstacle toe approach distances and vertical toe clearance heights among older adults are significantly lower than those demonstrated by young adults [2].

Older adults tend to react sooner to less severe balance disturbances than younger adults $[5,11]$. In addition, older adults demonstrate less sagittal plane COM motion, shorter crossing step length, smaller step width, slower crossing velocity, shorter postobstacle heel strike distance, and larger preobstacle toe approach distance than young adults during obstacle negotiation $[2,6,7]$. These findings suggest that older adults are at a higher risk for tripping during obstacle negotiation tasks due to the higher probability of obstacle contact enhanced by low clearance height.

One hypothesis for the increase of stepping among older adults is that older adults may be unable to gen- erate the necessary counterbalancing muscle torques, either in magnitude or in the rate of muscle torque development, needed to control the body's horizontal momentum [1, 12, 13]. However, this explanation should be viewed cautiously. Recent studies further reveal that, compared to young adults, limited frontal plane pelvic motion, kinetic accommodation in the sagittal plane, and nonoptimal foot placement might contribute to a greater risk of tripping in elderly adults $[2,36]$.

Stumbling responses in the elderly do not differ much from the responses of young adults. In several studies, both age groups demonstrated similar recovering strategies to perturbation [29, 49]. However, some important differences in the stumbling responses of older and young adults were observed. First, the elderly were less successful in avoiding the obstacle after tripping in early swing. Second, after both early and late swing perturbations, the characteristics of the motor responses, such as response latency and amplitude, varied between both groups [49]. The increased risk of falling after tripping in the elderly results from changed muscle responses in the stumbling reaction of older adults.

Older adults demonstrate ineffective early reactive postural responses due to slower onset latencies and smaller magnitudes of primary postural muscle responses which result in a longer time required to fully regain balance. A second indication of reduced effectiveness is demonstrated in a longer duration of coactivating both legs (perturbed and nonperturbed). This reflects increased energy expenditure required to regain balance. This response also slows down the restoration of joint trajectories to those present during normal walking. A secondary balance strategy observed in older adults is the activation of two posterior muscles on the nonperturbed leg (medial gastrocnemius and gluteus maximus). The activation of these two muscles may partially account for the shortened stride length after the slip.

It has also been suggested that recovery from tripping is partially dependent on controlling the resulting trunk flexion [22]. Individuals with greater trunk flexion during gait are less likely to regain control of their trunk before a point of terminal instability after tripping [45].

\section{Conclusion}

In conclusion, older adults are expected to have different moving patterns from younger adults expressed by differences in kinematics and kinetics of 
the limbs. The literature indicates that several biomechanical variables can be used to anticipate and prevent a potential fall. It is important to expand research methods to more realistic situations found in the elderly person's environment. Once one can predict who will fall as well as when and where that fall is most likely to occur, actions can be taken to prevent it.

The ultimate goal is to provide the older adult with an acceptable level of functioning. Conservation and restoration of muscular control and strength are important for optimum functioning. To achieve this goal, well-designed programs must be planned, taking into consideration dynamic stability research findings. The protocols must be decided on an individual basis because of the numerous variables that must be considered. Designing and applying an appropriate program to this population is not an easy task. Yet, in spite of the difficulties, there is no room for shortcuts. Regular participation in activities of moderate intensity should be encouraged in the older community to preserve physical functioning and independence. It is clear that the outcome of the vicious cycle-low performance level $\rightarrow$ sedentary habits $\rightarrow$ loss of function $\rightarrow$ lower performance levels—is dependency.

\section{References}

1. Angulo-Kinzler RM, Mynark RG, Koceja DM (1998) Soleus $\mathrm{H}$-reflex gain in elderly and young adults: modulation due to body position. J Gerontol Med Sci 53A:M120-M125

2. Begg RK, Sparrow WA (2005) Gait characteristic of young and older individuals negotiation a raised surface: implications for the prevention of falls. J Gerontol Med Sci 55A:M147-M154

3. Besser M, Selby-Silverstein L (2001) Predicting fall risk in the elderly using temporal-spatial parameters of gait. Symposium of the International Society for Postural and Gait Research, Maastricht, The Netherlands, Control of Posture and Gait, pp 70-73

4. Bohannon RW (1997) Comfortable and maximum walking speed of adults aged 20-79 years: Reference value and determinants. Age Aging 26:15-19

5. Brown LA, Shumway-Cook A, Woolacott MH (1999) Attentional demands and postural recovery: the effect of aging. J Gerontol Med Sci 54A:M165-M171

6. Chen HC, Ashton-Miller JA, Alexander NB, Schultz AB (1991) Stepping over obstacles: gait pattern of healthy young and old adults. J Gerontol Med Sci 46:M196-M203

7. Chen HC, Ashton-Miller JA, Alexander NB, Schultz AB (1994) Age effect on strategies used to avoid obstacles. Gait Posture 2:139-146

8. Craik RL, Dutterer L (1995) Spatial and temporal characteristics of foot fall patterns. In: Craik RL, Oatis CA (eds) Gait analysis, theory and applications. St. Louis, Mosby, pp 143-158

9. Cunningham DA, Rechnitzer PA, Pearce ME, Donner AP (1982) Determinants of self selected walking pace across ages 19 to 66. J Gerontol 37:560-564
10. Dargent-Molina P, Favier F (1996) Fall related factors and risk of hip fracture: the EPIDOS prospective study. Lancet 348:145-149

11. Deborah FV, Nichols JF, Turkel E, Hovell MF (1997) Effect of resistance, balance, and gait training on reduction of risk factors leading to falls in elders. J Aging Phys Act 5:213228

12. Dietz V (1992) Human neuronal control of automatic functional movements: interaction between central programs and afferent input. Physiol Rev 72:33-69

13. Dietz V, Quintern J, Boos G, Berger W (1986) Obstruction of the swing phase during gait: phase dependent bilateral leg muscle coordination. Brain Res 384:166-169

14. Donaldson MG, Khan KM (2002) Falls prevention starts with screening. Biomechanics 9:67-74

15. Draganich LF, Kuo CE (2004) The effects of walking speed on obstacle crossing in healthy young and healthy older adults. J Biomech 37:889-896

16. Elble RJ, Thomas SS, Higgins C, Colliver J (1991) Stridedependent changes in gait in older people. J Gerontol 238:1-5

17. Elizabeth TH, Robinovitch SN (1999) Biomechanical influences on balance recovery by stepping. J Biomech 32:1099-1106

18. Ferrandez AM, Pailhous J, Durup M (1990) Slowness in elderly gait. Exp Aging Res 16:79-89

19. Gabell A, Nayak USL (1984) The effect of age on variability in gait. J Gerontol 39:662-666

20. Gillis B, Gilory K, Lawley H, Mott L, Wall JC (1986) Slow walking speed in healthy young and elderly females. Physiother Canada 38:350-352

21. Grabiner MD (1997) Locomotion in healthy older adults. In: Allad P, Capozzo A, Lundberg A, Vaughan CL (eds) Threedimensional analysis of human locomotion. Wiley, Chichester, UK, pp 363-373

22. Grabiner MD, Koh TJ, Lundin TM, Jahnigen DW (1993) Kinematics of recovery from a stumble. J Gerontol Med Sci 48:M97-M102

23. Hahn ME, Li-Shan C (2004) Age-related reduction in sagittal plane center of mass motion during obstacle crossing. J Biomech 37:837-844

24. Hagemon PA, Blanke DJ (1986) Comparison of gait of young women and elderly women. Phys Ther 66:1382-1387

25. Horak FB, Shupert CL, Mirka A (1989) Components of postural dyscontrol in the elderly: a review. Neurobiol Aging 10:727-738

26. Hylton BM, Lord SR, Fitzpatrick RC (2003) Age-related differences in walking stability. Age Aging 32:137-142

27. Imms FJ, Edholm OG (1979) The assessment of gait and mobility in the elderly. Age Aging 8:261-267

28. Imms FJ, Edholm OG (1981) Studies of gait and mobility in the elderly. Age Aging 10:147-156

29. Jensen JL, Brown LA, Woollacott MH (2001) Compensatory stepping: the biomechanics of a preferred response among older adults. Exp Aging Res 27:361-376

30. Jevsevar SD, Riley PO, Hodge WA, Krebs DE (1993) Knee kinematics and kinetics during locomotor activities of daily living in subjects with knee arthroplasty and in healthy control subjects. Phys Ther 73:229-239

31. Krebs DE, Wong D, Jevsevar D, Riley PO, Hodge WA (1992) Trunk kinematics during locomotor activities. Phys Ther 72:505-514

32. Lord SR, Lloyd DG, Li SK (1996) Sensory-motor function, gait patterns and falls in community-dwelling women. Age Aging 25:292-299

33. Lu TW, Chen HL, Chen SC (2006) Comparisons of the lower limb kinematics between young and older adults when crossing obstacles of different heights. Gait Posture (in press) 
34. Maki BE (1997) Gait changes in older adults: predictors of falls or indicators of fear. J Am Geriatr Soc 45:313-320

35. Mbourou GA, Lajoie Y, Teasdale N (2003) Step length variability at gait initiation in elderly fallers and non-fallers, and young adults. Gerontology 49:2-6

36. McFadyen BJ, Prince F (2002) Avoidance and accommodation of surface height changes by healthy, communitydwelling, young and elderly men. J Gerontol 57A:B166B174

37. McGibbon CA, Krebs DE (2001) Age-related changes in lower trunk coordination and energy transfer during gait. J Neurophysiol 85:1923-1931

38. McKenzie NC, Brown LA (2004) Obstacle negotiation kinematics: age-dependent effects of postural threat. Gait Posture 19:226-234

39. Murray MP, Kory RC, Clarkson BH (1969) Walking patterns in healthy old men. J Bone Joint Surg Am 1969 24:169-178

40. Myers AH, Young Y, Langlois JA (1996) Prevention of falls in the elderly. Bone 1996 18:87S-101S

41. Oberg T, Karsznia A, Oberg K (1993) Basic gait parameters: reference data for normal subjects, 10-79 years of age. J Rehabil Res Dev 30:210-223

42. O'Brien M, Power K, Sanford S, Smith K, Wall J (1983) Temporal gait patterns in healthy young and elderly females. Physiol Can 35:323-326

43. Owings TM, Grabiner MD (2004) Variability of step kinematics in young and older adults. Gait Posture 20:2629

44. Patla AE, Winter DA, Frank JS, Prasad S, Walt SE (1989) Identification of age-related changes in the balance control system. In: Duncan PW (ed) Balance. American Physical Therapy Association, Alexandria, pp 43-55
45. Pavol MJ, Owings TM, Foley KT, Grabiner MD (1999) Gait characteristic as risk factors for falling from trips induced in older adults. J Gerontol 54A:M538-M590

46. Perry J (1992) Gait analysis. Normal and pathological function. SLACK, Thorofare, NJ

47. Riccio GE (1993) Information in movement variability about the qualitative dynamics of posture and orientation. In: Newell KM, Corcos DM (eds) Variability and motor control. Human Kinetics, Champaign, IL 317-357

48. Scarborough DM, Krebs DE, Harris BA (1999) Quadriceps muscle strength and dynamic stability in elderly persons. Gait Posture 10:10-20

49. Schillings AM, Mulder TH, Duysene J (2005) Stumbling over obstacle in older adults compared to young adults. J Neurophysiol 94:1158-1168

50. Sudarsky L (1990) Geriatrics: gait disorder in the elderly. N Engl J Med 322:1441-1446

51. Tinetti ME, Speechley M, Ginter SF (1988) Risk factors for falls among elderly persons living in the community. N Engl J Med 319:1701-1707

52. Wagenaar RC, Kenneth GH, Kubo M, Chia-Ling H (20022003) Gait risk factors for falls in older adults: a dynamic perspective. Generations 26:28-32

53. Winter DA (1995) A.B.C (anatomy, biomechanics and control) of balance during standing and walking. Waterloo Biomechanics, University of Waterloo

54. Winter DA, Patla AE, Frank JS, Walt SE (1990) Biomechanical walking pattern changes in the fit and healthy elderly. Phys Ther 70:340-347

55. Woolacott MH, Tang PF (1997) Balance control during in the older adults: research and its implication. Phys Ther 77:646-660 